\title{
Marbling Grading Framework Applied on Meat Boutique Environment
}

\author{
Saulo Martiello Mastelini \\ State University of Londrina \\ (UEL) \\ Computer Department \\ Londrina, Brazil
saulomastelini@gmail.com matheuscmilo@gmail.com
}

\author{
Ana Paula Ayub da Costa \\ Barbon \\ State University of Londrina \\ (UEL) \\ Department of Animal Science \\ Londrina, Brazil \\ apbarbon@gmail.com
}

\author{
Sylvio Barbon Jr. \\ State University of Londrina \\ (UEL) \\ Computer Department \\ Londrina, Brazil \\ barbon@uel.br
}

\begin{abstract}
Bovine meat commercialization has an important role in the general food market scenario. The beef quality evaluation is realized through many ways, being one of the parameters the intramuscular fat amount (marbling). This evaluation is often made by a visual approach, so the process is subjective and susceptible to some errors sources. The use of Computer Vision techniques results in an automatized, non-subjective, fast and accurate method for evaluation. This paper presents the modeling and development of a Computer Vision System for Marbling evaluation, applied on a meat Boutique, localized in Londrina - PR. The proposed System uses a Computer Vision approach to control the features of the marbling analysis tool, aiming to satisfy sanitary requirements for non-contamination of the analyzed samples. Besides that, multiples samples on the scene are supported by our application. The proposed Computer Vision System has proved to be suitable for implantation in a production environment, like a meat Boutique.
\end{abstract}

\section{Categories and Subject Descriptors}

I.4.0 [Image Processing and Computer Vision]: General-Image Processing Software; I.4.6 [Image Processing and Computer Vision]: Segmentation-Pixel classification; I.4.8 [Image Processing and Computer Vision]: Scene Analysis - Object recognition; I.4.9 [Image Processing and Computer Vision]: Applications; J.7 [Computer Applications]: Computer in Other Systems-Command and control, Consumer products, Real time

Permission to make digital or hard copies of all or part of this work for personal or classroom use is granted without fee provided that copies are not made or distributed for profit or commercial advantage and that copies bear this notice and the full citation on the first page. To copy otherwise, to republish, to post on servers or to redistribute to lists, requires prior specific permission and/or a fee.

SBSI 2016, May 17th-20th, 2016, Florianópolis, Santa Catarina, Brazil Copyright SBC 2016.

\section{General Terms}

Computer Vision

\section{Keywords} lity

Computer Vision, Segmentation, Beef, Image, Food Qua-

\section{INTRODUCTION}

Brazil has a significant role in the global food industry and it is the largest beef exporter in the world. However, research into better standards and meat quality evaluations are essential for maintaining this status in the global food industry. The Marbling analysis is one of the various evaluations of meat quality. Marbling is caused by the visual intramuscular distribution of fat and it is one of the main attributes of meat. These attributes determine consumer's choice when purchasing because they influence the palatability, texture, and tenderness of the meat [11].

The traditional approach is performed by a trained specialist, called panelist, and it can be influenced by visual criteria since it is conducted by visual comparison with photographic standards [19]. This assessment is the assignment of a marbling score according to the known photographic pattern that goes from values 1 to 10 , as the amount of visible fat present in muscle. The higher the score, the greater the amount of fat [2].

For panelists, the marbling visual association of a score is influenced by visual interpretation subjectivity. This problem is due to poor repeatability and strong influence of the environment on evaluators, besides being a lazy evaluation, costly and tedious $[4,14,7]$.

In recent years, several studies have addressed the possibility of meat quality evaluation based on processes involving computing. Various computer vision techniques and machine learning technologies have been applied to meat quality classification $[4,8,16,13]$.

In [4], a method for meat marbling segmentation is developed, using a region growing algorithm and a pixel of the 
lean portion of the sample as a seed for this procedure. The chosen color space channel is the $\mathrm{L}^{*} \mathrm{a} * \mathrm{~b} *$ Luminance component. The authors compare the proposed method with techniques of segmentation consolidated on literature and they achieve better results on tests.

A method for automatic segmentation of beef image samples is proposed on [8]. The developed methodology uses empiric threshold values, a clustering algorithm and small regions removing techniques. The authors get satisfactory results, but specular reflexion is a problem that the proposed method does not solve. Also, the papers $[10,9]$ present methods to evaluate and segment beef marbling areas.

Several works on meat quality assessment are presented on [17], using Computer Vision (CV) as solution. Besides that, in the book [5] some CV methods are applied on meat quality evaluation.

Many of the related works achieve satisfactory results in the marbling segmentation task $[8,10,9]$, but some points need to be addressed. The image acquiring systems used in these methodologies have controlled environments with uniform artificial incident luminosity to capture the pictures, which are not suitable for production routines and environments. Some parameters used in the related method are determined empirically, so these algorithms are appropriate for a specific scenario. A later point is the control system strategy. None of the related papers have a methodology for controlling the features of their systems in conformity with sanitary requirements. The solutions are laboratory approaches and unsuitable for production environments.

Thus, the aim of this paper was to propose a more objective framework to determine the marbling level in beefs using digital imaging and computing, specifically a Computer Vision System (CVS), to be applied in a real production environment and handling meat. The chosen environment was a meat Boutique, a place where high quality and cost meat is commercialized, which reinforced the need for an accurate method without contact with the samples

This paper is organized as follow: Section 2 presents the scenario of implementation of the proposed Framework. Section 3 presents the proposed solution, highlighting its use cases, main components, methodologies, and technologies employed. Section 4 describes the implantation tests used to validate the Framework features. Section 5 presents the a study case of implantation of our Framework and the building of a model based on the Boutique environment and meat samples for marbling grade. Finally, Section 6 presents the final considerations of this paper, the possible future work and researches related to the proposed solution.

\section{MEAT BOUTIQUE SCENARIO}

The environment for application of the proposed framework was a meat Boutique located in the city of Londrina (Paraná - Brazil). In this place, high-value meat pieces are commercialized, including beef, in which higher intramuscular fat score (marbling) increases their commercial value.

Previously to the use of a CVS solution, the traditional method to evaluate meat marbling in the Boutique was made in live animals. On select animals, a professional measures general marbling grade using an ultrasonography device. This technique is known to be non-invasive and has an accurate estimation of carcass composition for sorting carcasses on quality. The images are obtained from one animal at a time and provide information on the percentage of intramus- cular fat, ribeye area, and fat thickness. However, it has a high cost compared to other techniques that evaluate several samples. $[1,12]$. In this aspect, at the time of sale, an expensive meat may be sold for a lower price because of the "global" marbling grade on this particular animal. Moreover, this approach depends on a subjective evaluation of a trained specialist.

Another consolidated approach is the employment of trained specialists, which using an image pattern of marbling grade, classify each meat sample using a visual evaluation approach with the assignment of a score to the meat pieces.

Considering the higher price of meat sold in a place like a meat Boutique, an accurate evaluating method is essential to give the real marbling grade on each beef sample and thus maximize profits.

Moreover, the characteristics of the product bring a set of sanitary factors to be achieved, avoiding contact of any material with the samples, and thus preventing contamination. For this reason, the meat cut room on Boutique is an isolated area where only authorized personnel can enter, using appropriate clothing, like boots, gloves, and cap.

\section{PROPOSED SOLUTION}

The developed system uses a camera for scene capture, object detection and automatic segmentation of marbling on the meat samples images.

For proposed solution development we used Java and OpenCV. Regarding the hardware components, we aim for cost reduction without compromising performance and reliability. In this aspect, a Full HD webcam camera (4tech PK-910H, $1080 \times 1920$ resolution) was chosen. Also, we used a home personal computer (4GB of RAM, Core i5 2nd generation processor) to run the System software.

Figure 1 presents a general overview of our Framework, highlighting its main hardware components.

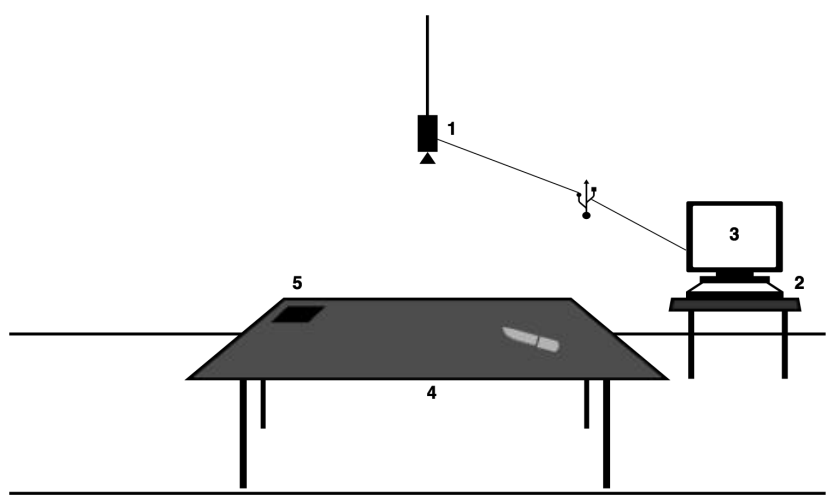

Figure 1: General overview of proposed Framework components: 1) Webcam, 2) Computer, 3) Computer screen, 4) Cutting table, and 5) Marker

As showed in Figure 1, the main components of our Framework are a Webcam camera (1), placed on the Cutting Table (4), where multiples samples of meat should be handled and sliced. Also, a Computer (2) running the developed software and a screen (3) for visual feedback of system manipulation are components of the Framework. A marker (5) is placed in a corner of the cutting table. The System recognizes this object in the scene captured by the camera and 
trigger an action. This choice for controlling the features of the proposed Framework was motivated by sanitary requirements of non-contamination of meat, that may be caused by contact with unsterilized objects. Thus, the operator of the System can not touch any controlling surface, like buttons, mouse or keyboard.

Figure 2 presents a diagram of defined markers placement and the control action related to each one. The placement of a square marker in one of the corners trigger an Framework action. The Framework control will be discussed in more detail in the next sections.

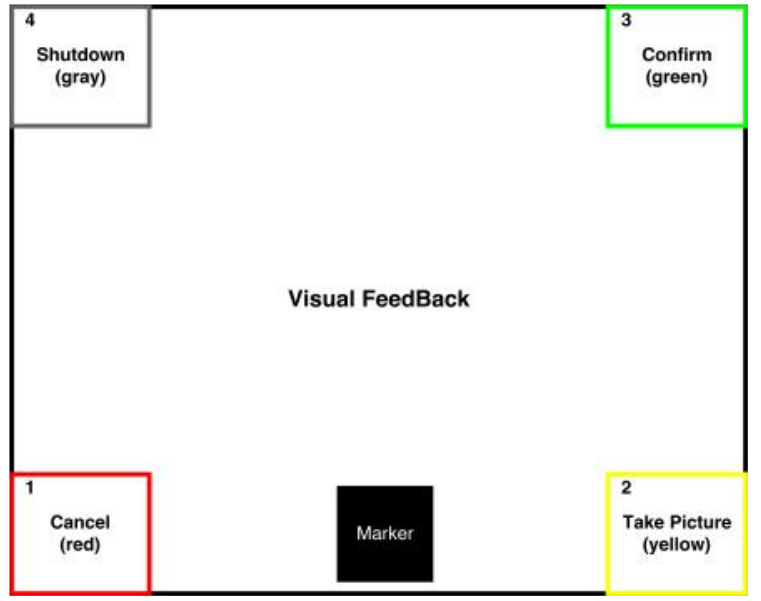

Figure 2: Corners configuration and actions triggered

The following sections describe the methodology used for the creation of the Framework for automated marbling detection and rating. First, we present the use cases of our Framework, next the approaches for Framework control, meat samples recognition and marbling segmentation. Finally, to build a model for mapping the intramuscular fat to a marbling grade, a linear model is presented, which was done using an interpolation between the scores gave from a trained panelist and the percentage values from the marbling segmentation algorithm, obtained after a period of sampling process on the meat Boutique.

\subsection{System Operation}

The proposed Framework aims to offer a simple control solution for marbling evaluation, hence the interface for user allows only a few (necessary) controlling operations.

The basic operation takes a photo and evaluates marbling over the meat samples on the scene. For this purpose, with samples over the cutting table, the Operator (User) places the marker on the yellow corner (corner 2). The Framework recognizes the marker, it takes a picture and freezes the image on screen feedback. The Operator observes the taken picture and chooses between two options: cancel and discard the actual frame or confirms and let the Framework proceed on marbling evaluation over the captured frame. The former is achieved placing the marker over the red corner (corner 1 ), which means the cancel operation; the latter corresponds to place the marker on the green corner (corner 3), and it means the confirm operation.

After the boot of computer and the automatic start of the proposed Framework, the Operator must not touch any buttons or another kind of contact controlling inputs. So, to finishes the meat analysis and shutdown the System, a marker command is used. The Operator places the marker on the gray corner (corner 4), and the System recognizes this action. The Operator chooses one of two options: cancel (place the marker on the red corner) or confirms and shutdown the System (placing the marker on the green corner).

In Figure 3 the general information flow of our proposed tool is presented.

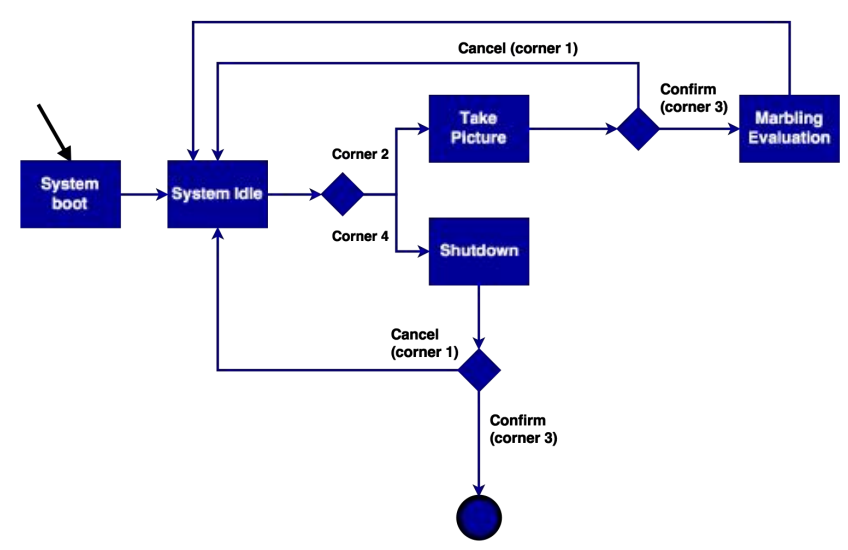

Figure 3: General overview of proposed Framework flow

\subsection{Framework Control}

Due to sanitary factors, the designed Framework must not require physical contact for operation. Touching any unsterilised surface can contaminate the manipulated meat. In this aspect, the controls of the proposed tool had to be designed using commands captured by the camera. We used markers recognition in the scene to control the Framework.

The strategy of markers recognition was the shape of the marker. Square markers $(5 \times 5 \mathrm{~cm})$ were adopted to avoid extra processing and time delay on control, due to its form simplicity. Determined regions in the picture (corners) were chosen to be mapped at regular intervals and in these subimages the presence of square markers has come to represent a control action in the Framework, as described in Figure 2. The markers detection was done as follow.

First, the sub-image was converted to grayscale and transformed into a binary representation through the Otsu thresholding technique [15]. Using a tregion growing algorithm (OpenCV floodfill method) all regions touching the borders of the image were removed. Thereby, only square objects entirely located inside the picture were detected.

After that, all contours in the image were identified (through of findCountours OpenCV method) and over each one of these shapes four properties were evaluated: the number of sides, region size, aspect ratio and solidity.

A square shape must have four sides, but due to image distortion and noise, contours with the number of sides between four and six were taken into account (first property). A marker should fill a relevant portion of the scene, so smaller contours regions than $0.1 \%$ of total image size were removed (second property). Besides that, the aspect ratio of a square, in other words, the ratio between height and weight of a square is equal to one, since all its sides have the same length. In our Framework, we considered candidates to 
markers contours with the aspect ratio between 0.8 and 1.2 (third property). A convex hull is the smaller set of points where all lines connecting two points inside of this set are contained inside the group [6]. The solidity of a geometric shape is the ratio between its area and the area of its convex hull. Squares have a solidity of one, but in our approach, we considered regions with solidity greater than 0.9 (fourth property).

If the properties mentioned are satisfied, the analyzed contour is considered a marker and the described routine will finish.

\subsection{Meat Samples Recognition}

There are different segmentation methods that could theoretically be used for object (meat) recognition on an image, methods such as Circle Hough Transform. This method is capable of recognizing an object by its shape. Hough's algorithm locates a possible border with the help of a canny edge detector. From the perpendicular lines to the edge points derivatives, it determines the center regions, as well the borders regions, and as a result, the object itself [20].

However, due to high computational costs and an unacceptable segmentation error rate, it was discarded for the Watershed segmentation method, which presents a fast processing speed, allowing it to be used on interactive applications, even when the processing images are fairly large and complex [18], besides showing acceptable segmentation error rates. Besides that, in the scenario of implantation of our Framework, multiple samples should be on the scene, and some of them may be overlapping. This fact motivated the usage of the Watershed algorithm since it can solve this situation, splitting the overlapped samples.

There are two basic ways of working with segmentation, the first one is based on borders and shapes to recognize objects, for instance, the Circle Hough Transform algorithm. While the second detects similarities between pixels and regions, as in the Watershed algorithm [3].

The Watershed segmentation method calculates the gradient for all pixels from the image. The segments were formed by regions that by sharing a local minimum, indicate an object of interest. The arrangement of the pixels was made by search mechanisms of close values from each local minimum [18], ideal for segmentation of objects regardless of their shape.

This method allows the segmentation of objects, requiring only the marking of three types of regions with different tones of color: the sure background region, the sure foreground (object of interest) region, and finally the uncertainty regions, where is not known where each pixel belongs to. The Watershed algorithm finds the borders of the objects of interest through an affinity analysis of the marked colors of the region, in a similar way to the shed of water on a topographic map [3], naming the process. The result is the object's segmentation.

Figure 4 presents the application of Watershed algorithm on an image taken on implantation the environment. On Figure $4 \mathrm{a}$ the original image is presented. Figure $4 \mathrm{~b}$ presents the resulting image after watershed application.

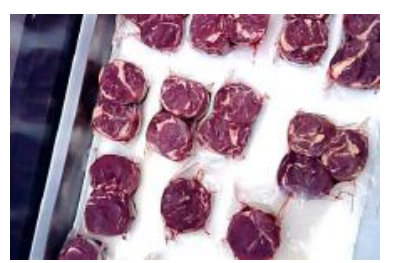

(a)

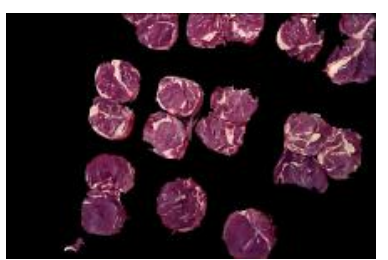

(b)
Figure 4: Watershed application over meat samples image. a) original image and b) result of watershed application

We used the Otsu thresholding technique [15] over the H channel of HSV color space for background removal before the Watershed algorithm application.

\subsection{Marbling Segmentation}

With a meat sample image given from Watershed algorithm described in Section 3.3, next step is marbling segmentation from image. To separate meat from the background the strategy adopted was take the result of B minus $\mathrm{G}$ from RGB color space. On this resulting grayscale image, the Otsu thresholding method was applied resulting in a primary meat mask. Small connected regions were removed from mask through of a region growing algorithm (OpenCV floodFill), using as threshold $0.3 \%$ of image size. To avoid extra processing, the image was cropped, so only a rectangular area around the beef sample was kept.

Before the segmentation of marbling, many of the meat pieces had a fat surrounding layer. This fat amount must not be evaluated as marbling. So, this covering fat content must be removed from the mask. To do that, first the actual meat mask was applied on original image. We took an HSV color space representation from this new image. All whitish regions, in other words, fat regions, were computed through the application of Otsu technique over a V (Value) minus $\mathrm{S}$ (Saturation) image. In this aspect, using the HSV image, a new mask with only fat was created. A copy of this mask was made, and it was subjected to a NOT logical operation.

At this point, the algorithm originated three masks: the beef mask, the fat regions mask, and the mask resultant from described NOT operation. The latter represents all the nonfat areas plus the background. A new mask only with meat results from a $A N D$ operation between the non-fat mask and the beef mask was created. Next step was the removal of the covering fat layer properly speaking. Thereby, an opening morphological operation was applied on fat mask to separate few connected regions. This procedure was necessary since the fat layer may be connected with marbling regions. We used the same floodFill region growing algorithm to remove white content greater than $0.2 \%$ of image size. The result was a rough marbling mask. The fat covering layer removal was completed with a $O R$ logical operation between the marbling mask and the meat mask.

Again the floodfill method was applied filling the holes on the mask. To ensure that all surrounding fat is removed, the erode morphological operation was used. The segmentation of marbling was finally done using the same $\mathrm{V}$ minus $\mathrm{S}$ approach, with Otsu thresholding technique. The last step was to compute the percentage of marbling over the whole beef area.

Figure 5 shows the main steps of the marbling segmenta- 
tion process. In Figure 5 a the result of background removal and image crop is presented. The removal of the surround fat layer of beef is presented in Figure 5b. The result of application of the erode morphological operation is presented on Figure 5c. Lastly, Figure 5d presents the result of marbling segmentation process.

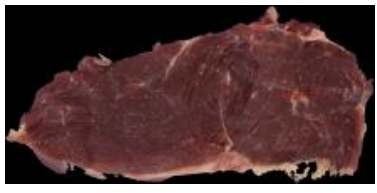

(a)

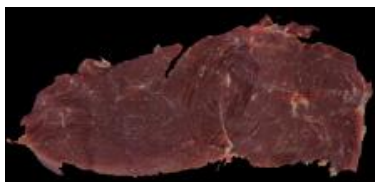

(c) (b)

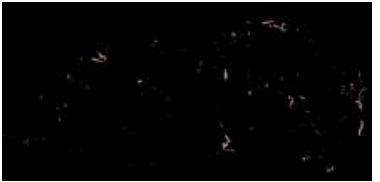

(d)

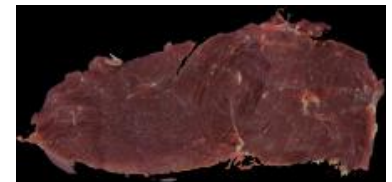

Figure 5: Segmentation main steps: a) Sample after background removal and crop, b) External fat layer removal, c) Morphological erode and d) Marbling segmentation

\subsection{Regression Model}

Our segmentation algorithm returns a percentage value corresponding to the amount of intramuscular meat content. However, how previously described, the marbling classification is done through levels (marbling grade) and not fat amount percentage. In this aspect, it was necessary to build a model for grading the meat, transforming the value returned by the segmentation method to a marbling level. We chose a linear model to do this mapping.

The modeling consists of taking the percentage value of our segmentation method ( $x$ axis) and a marbling grade coming from a trained panelist ( $y$ axis), for some meat samples. Joining the measured values of each sample as a Cartesian coordinate, it is possible to draw a line (linear function), which approximately cover all points in the bi-dimensional space, relating the two quantities. After that, given a percentage value, it is possible to determine a marbling grade using this function.

Due to factors like camera quality and distance to the samples, environment conditions and reflectance over meat surface, the percentage value given by the segmentation algorithm may differ from place to place. Thereby, it is necessary to build a different regression model for a different sampling environment.

After the linear model definition, in the production-line, all percentage values coming from the segmentation algorithm must be subjected to the described function, returning a marbling grade level. If the built model has a high correlation $(R)$ between $x$ and $y$ components, then the function is a good representation of the behaviour of the observed increasing/decreasing pattern.

\subsection{Overview of Marbling Grading Strategy}

The general flow of marbling grading strategy of our Framework is presented in Figure 6. As described in the figure, firstly an image of cutting table is taken (1). Next, this image is subject to Watershed algorithm for background removal and meat samples segmentation (2), generating a meat sample image list (3). If this list is not empty, each image contained inside the structure is subjected to the Marbling Segmentation procedure (4). The latter generates a percent value, corresponding to the amount of visible intramuscular fat. The mentioned percent value is applied on the marbling score regression model, generating a Marbling grade (5). This grade is written over the corresponding sample in original taken image (6). After all images in meat sample list were processed the modified image with the marbling grades is showed in the Graphic User Interface.

These procedures are executed after every image capture action on Framework.

\section{VALIDATION AND IMPLANTATION}

Initially, tests of control and functionality were executed during and after the development period. The markers recognition and Framework control were tested over a common flat table, aiming for reproduce the meat Boutique cutting table. We used printed square markers for controlling of the features of the System.

The beef recognition, segmentation and marbling evaluation features were validated by testing using beef images photos. On these images, we removed the background manually keeping only beef. After that, the cropped images were positioned in many configurations over the same table earlier mentioned. The webcam was positioned above the table using a temporary support device and it was connected to a notebook, running the software of developed Framework. All the functions of the designed tool were tested and validated using this simulated environment.

Figure 7 shows a screen capture of Framework software running during a laboratory test of our proposed tool. On this scene, a cropped image of beef is on the test table, and after the capture action was performed, the control marker is placed over the confirmation corner, signalizing the beginning of the marbling segmentation process. Worth mentioning the rectangle around the meat picture, the result of the Watershed meat recognition procedure.

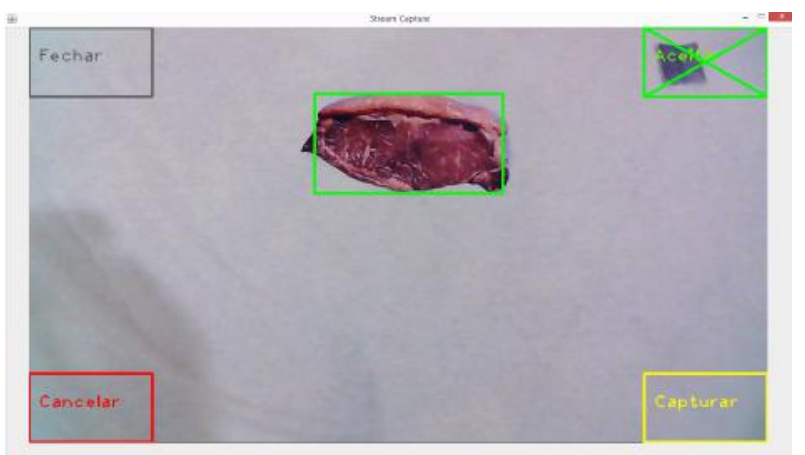

Figure 7: Framework screen capture during System tests

The environment for implantation was an isolated room for cutting the pieces of meat. So, the placement of camera to acquire images must not interfere with meat cut and packing process. Also, a screen for visual feedback must be in the room. For these reasons, a study of implantation was 


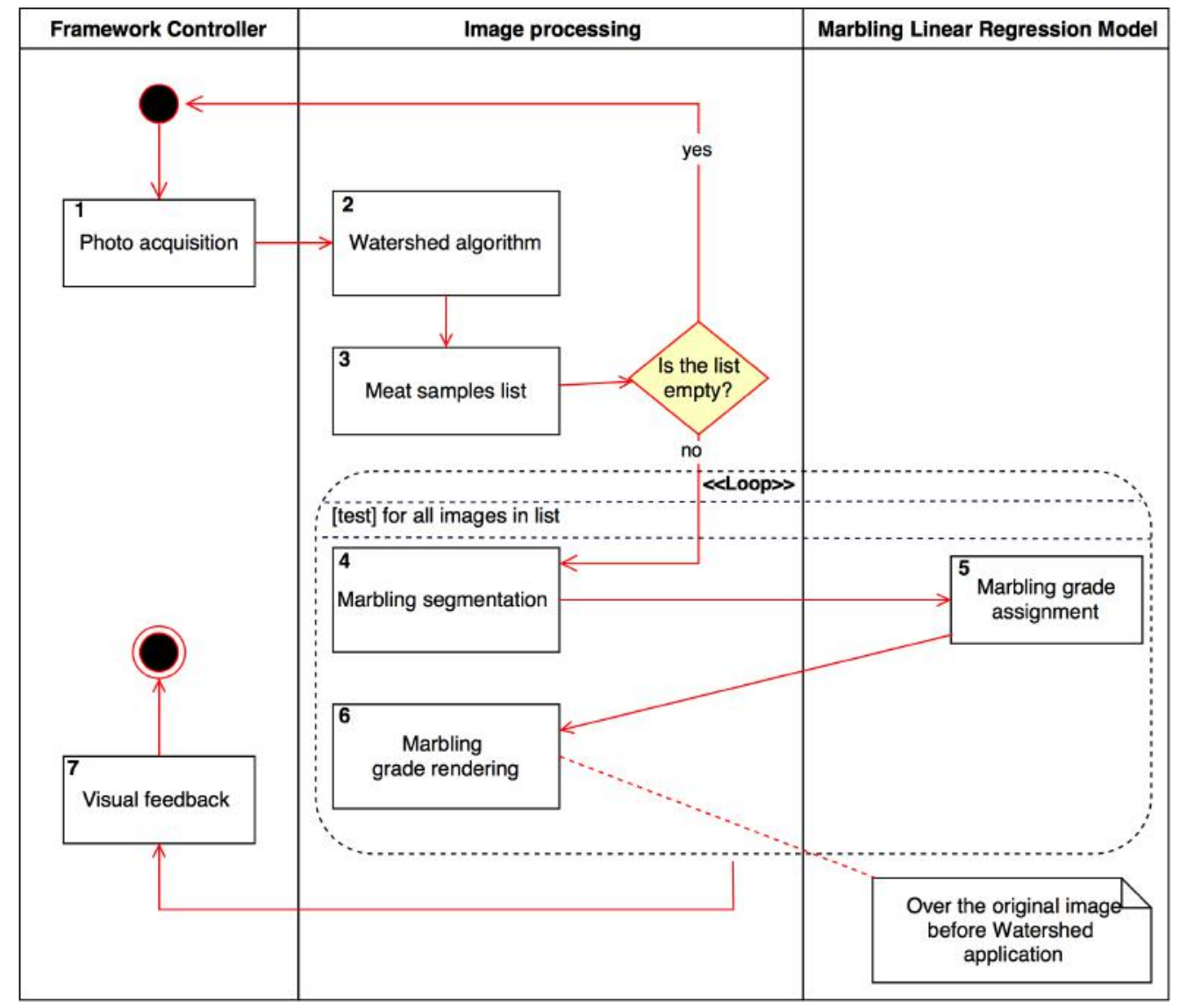

Figure 6: Marbling grading sequence diagram

realized.

The position of the camera may affect the whole image acquisition process since the angle of illumination between the camera and observed object is decisive to get adequate image sampling or images with excessive spectacular reflection.

All aspects mentioned were taken into account aiming for the better Framework component distribution inside the meat cutting room.

\section{STUDY CASE}

A study case of implantation of our Framework was performed on meat Boutique environment. Using the previously described hardware components, the System was put on running in the meat cutting room of the application scenario. A total of $n=6$ meat samples were used for Framework prototype and the marbling grade function building.

As described on Section 3.5 each analysed meat sample was classified by a trained panelist, using the American Meat Science Association (AMSA, 2001) pattern for marbling grading, and then submitted to our Framework. Thereby, to each analysed sample the grade level (trained panelist) and percentage intramuscular fat amount (Framework) were stored.

Figure 8 shows the samples used in the tests, after the meat recognition procedure, image cropping and background removal.
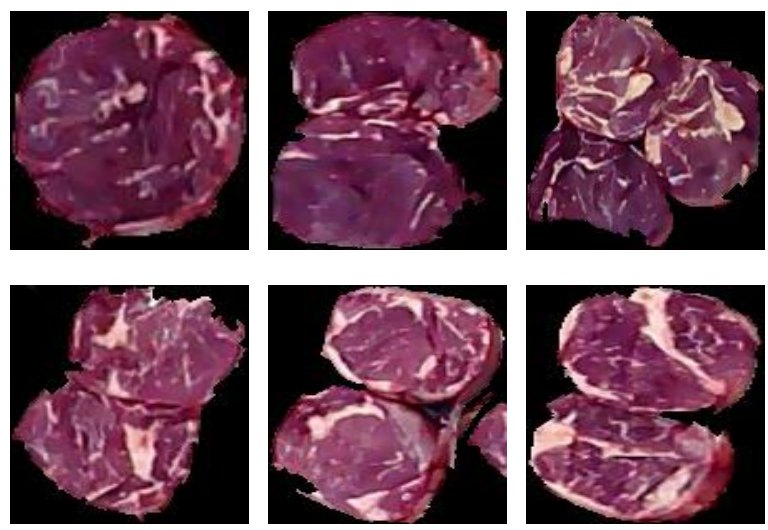

Figure 8: Samples used in tests (from left top to bottom right): images $1,2,3,4,5$ and 6

Table 1 shows to each analysed sample the marbling percentage given by our segmentation algorithm (middle column) and marbling grade given by the trained panelist (right column). Three classes of marbling grade were detected in the analysed samples. In fact, on the testing en- 
vironment high-quality meat is commercialized, hence the used samples reflect the products marbling grade sold in this place, since meat pieces with grades greater than three were observed.

\begin{tabular}{ccc}
\hline Sample & Framework & Panelist Grade \\
\hline 1 & $2.3 \%$ & 4 \\
2 & $1.7 \%$ & 4 \\
3 & $7.1 \%$ & 6 \\
4 & $7.3 \%$ & 7 \\
5 & $5.6 \%$ & 6 \\
6 & $5.3 \%$ & 6 \\
\hline
\end{tabular}

Table 1: Marbling percentage from proposed Framework and panelist marbling grade

After, a linear model was built using these values. Therefore, for the Boutique specific testing environment, a linear function mapping a percentage value to a marbling grade level was determined.

The resulting linear function was

$$
y=3.08+49.31 * x
$$

where $y$ is the marbling grade and $x$ is the detected marbling percentage on a sample.

The built model reaches a correlation of $R=0.9571$, which shows that the described linear function is a good representation of the relationship between marbling percentage and marbling panelist grade.

The correlation result shows that the proposed marbling analysis approach is suitable for implantation in a place like a meat Boutique. Besides that, the Framework controlling tests showed that the markers recognition strategy is an adequate choice for the System features operation. The control action is simple, easy for learning and satisfy the sanitary requirements that a meat handling environment requires.

\section{CONCLUSION}

Marbling is a parameter for meat quality evaluation. The traditional approach for marbling grading is done through visual inspection. This method is very subjective, tedious, susceptible to fatigue and environment influences. Another approach, applied on live animals is the use of a ultrasonography device for a general marbling panorama. This evaluation is very costly and gives only a marbling grade per animal, disregarding specific portions of the carcass, which may lower profits at sale time, due to an erroneous global grading method. Besides that, this global score depends on a specialist visual approach analysis, bringing the same disadvantages previously described.

The use of a CVS offers a cheap, fast, non-destructive, precise and robust alternative for the marbling evaluation task. In this paper, we presented the modelling and development of a Framework for beef marbling evaluation. The proposed solution deals with multiple samples in the same scene, evaluating separately the meat pieces, and giving a marbling grade for each sample. The tests originated satisfactory results for controlling the Framework, recognizing the samples and a high correlation between the percentage value from our marbling segmentation algorithm and the evaluation of a trained panelist.
As next step, the built marbling model should be validated using more samples for evaluation. Therefore, a period of testing will be determined by implantation and using of our System. During this period, the constructed linear model will be put on test, comparing the marbling given by our Framework and the panelist approach. The data analysed will be stored for possible future modifications and improvement of the proposed model.

\section{REFERENCES}

[1] D. E. Anderson and M. Rings. Current veterinary therapy: food animal practice. Elsevier Health Sciences, 2008.

[2] A. M. S. Association et al. Meat evaluation handbook. American Meat Science Association National Cattlemen's Beef Association (US) National Pork Producers Council (US), 2001.

[3] S. Beucher et al. The watershed transformation applied to image segmentation. SCANNING MICROSCOPY-SUPPLEMENT-, pages 299-299, 1992.

[4] K. Chen and C. Qin. Segmentation of beef marbling based on vision threshold. computers and electronics in agriculture, 62(2):223-230, 2008.

[5] Y. Cho and S. Kang. Emerging Technologies for Food Quality and Food Safety Evaluation. Contemporary Food Engineering. CRC Press, 2011.

[6] R. C. Gonzalez and R. E. Woods. Digital Image Processing (3rd Edition). Prentice-Hall, Inc., Upper Saddle River, NJ, USA, 2006.

[7] H. Huang, L. Liu, M. Ngadi, and C. Gariepy. Prediction of pork marbling scores using pattern analysis techniques. Food Control, 31(1):224-229, 2013.

[8] P. Jackman, D.-W. Sun, and P. Allen. Automatic segmentation of beef longissimus dorsi muscle and marbling by an adaptable algorithm. Meat Science, 83(2):187-194, 2009.

[9] P. Jackman, D.-W. Sun, P. Allen, K. Brandon, and A.-M. White. Correlation of consumer assessment of longissimus dorsi beef palatability with image colour, marbling and surface texture features. Meat Science, 84(3):564 - 568, 2010.

[10] P. Jackman, D.-W. Sun, C.-J. Du, and P. Allen. Prediction of beef eating qualities from colour, marbling and wavelet surface texture features using homogenous carcass treatment. Pattern Recognition, 42(5):751 - 763, 2009.

[11] K. Killinger, C. R. Calkins, W. J. Umberger, D. M. Feuz, and K. M. Eskridge. Consumer sensory acceptance and value for beef steaks of similar tenderness, but differing in marbling level. Journal of Animal Science, 82(11):3294-3301, 2004.

[12] N. Lambe, D. Ross, E. Navajas, J. Hyslop, N. Prieto, C. Craigie, L. Bünger, G. Simm, and R. Roehe. The prediction of carcass composition and tissue distribution in beef cattle using ultrasound scanning at the start and/or end of the finishing period. Livestock Science, 131(2):193-202, 2010.

[13] D. Liu, H. Pu, D.-W. Sun, L. Wang, and X.-A. Zeng. Combination of spectra and texture data of hyperspectral imaging for prediction of $\mathrm{pH}$ in salted meat. Food chemistry, 160:330-337, 2014. 
[14] L. Liu, M. Ngadi, S. Prasher, and C. Gariépy. Objective determination of pork marbling scores using the wide line detector. Journal of Food Engineering, 110(3):497-504, 2012.

[15] N. Otsu. A threshold selection method from gray-level histograms. Systems, Man and Cybernetics, IEEE Transactions on, 9(1):62-66, 1979.

[16] S. Sharifzadeh, L. H. Clemmensen, C. Borggaard, S. Støier, and B. K. Ersbøll. Supervised feature selection for linear and non-linear regression of $\mathrm{L}^{*} \mathrm{a}^{*} \mathrm{~b}^{*}$ color from multispectral images of meat. Engineering Applications of Artificial Intelligence, 27(0):211 - 227, 2014.

[17] D. Sun. Computer Vision Technology in the Food and Beverage Industries. Woodhead Publishing Series in Food Science, Technology and Nutrition. Elsevier Science, 2012.

[18] A. v. WANGENHEIM et al. Seminário introdução à visão computacional. Visão Computacional-Aldon von Wangenheim's HomePage, 2001.

[19] Z. Xiong, D.-W. Sun, X.-A. Zeng, and A. Xie. Recent developments of hyperspectral imaging systems and their applications in detecting quality attributes of red meats: A review. Journal of food engineering, 132:1-13, 2014.

[20] H. Yuen, J. Princen, J. Illingworth, and J. Kittler. Comparative study of hough transform methods for circle finding. Image and vision computing, 8(1):71-77, 1990. 